\title{
Simple Pre-Steering Constraints for Wideband LCMV-beamformer
}

\author{
Alexey A. Erokhin, Yury P. Salomatov, Evgeniy R. Gafarov \\ Institute of Engineering Physics and Radio Electronics \\ Siberian Federal University \\ Krasnoyarsk, Russia \\ AErokhin@sfu-kras.ru
}

\author{
Victor N. Ushakov \\ Faculty of Radio Engineering and Telecommunications \\ Saint Petersburg Electrotechnical University "LETI" \\ Saint Petersburg, Russia
}

\begin{abstract}
The LCMV-beamformers are widely used in many applications but to steer array pattern in some direction we need to use phase shifters or additional delay lines for presteering. In this paper, we introduce simple constraints in the DOA of the useful signal. These constraints have a form of a block-diagonal matrix. In a case when DOA is the broadside, simple constraints are equal to Frost's constraints.
\end{abstract}

Keywords - LCMV, Frost beamformer, adaptive antenna array, constraint matrix, wideband beamforming

\section{INTRODUCTION}

Linearly constrained minimum variance (LCMV) criterion is widely used in broadband adaptive antenna arrays that are described in many papers [1]-[4]. This criterion and iterative algorithm were proposed by Frost [5]. LCMVbeamformer uses tapped delay lines to increase broadband properties of the adaptive array. The adaptive antenna array is shown in Fig. 1, where $M$ is the number of the array elements, $\mathrm{J}$ is the number of taps in the delay lines.

In the Frost's algorithm, it is assumed that directional of arrival (DOA) of useful signal is the broadside. If DOA is different from the broadside, the array can be pre-steered towards the desired DOA. Pre-steering can be done electrically or mechanically. It is necessary to use phase shifters or additional delay lines [2], [6]. Some authors modify constraint matrix to avoid pre-steering: the form of constraint matrix is not a block-diagonal one [7]-[9]

In this paper, it is proposed to use simple constraints in the DOA of the useful signal in the form of a block-diagonal matrix.

The paper is organized as follows: Section II briefly introduces LCMV-solution in direct and iterative forms, Section III shows that we can use simple DOA constraints instead of pre-steering. In Section IV we discus simulation results. Section V includes some concluding remarks about the proposed constraints.

\section{LCMV (FROST'S) BEAMFORMER}

According to Fig. 1, the output array signal is:

$$
y(k)=\mathbf{w}^{H} \mathbf{X}(k),
$$

where symbol $(\bullet)^{\mathrm{H}}$ denote Hermitian transpose and bold symbols denote vectors and matrices.
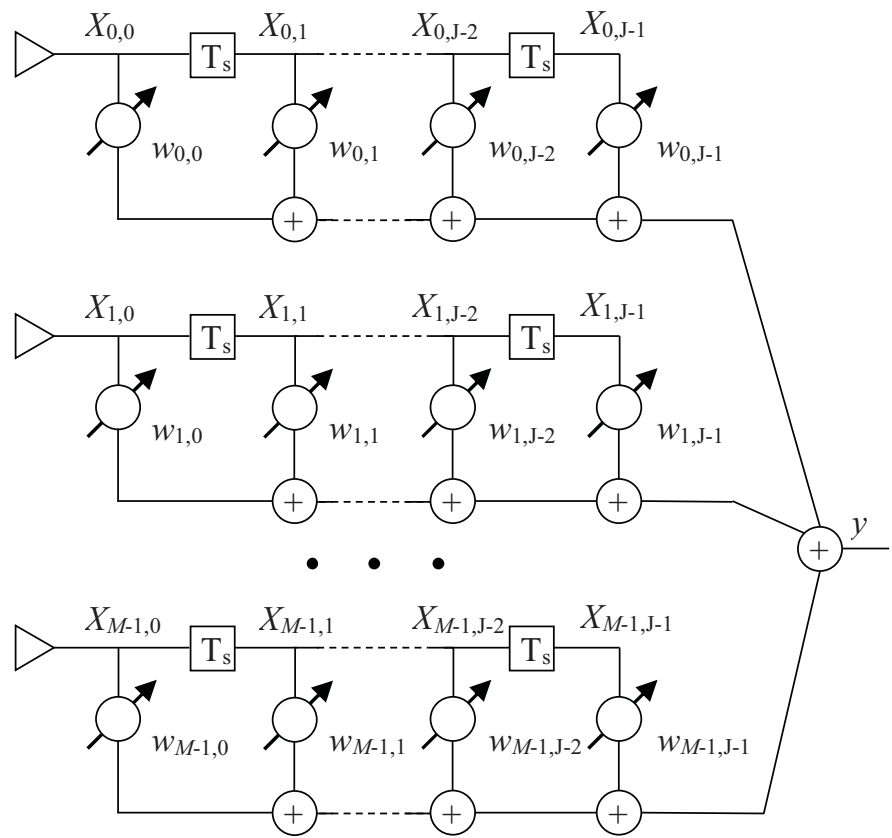

Fig. 1. Adaptive antenna array

The total array output power (variance):

$$
E\left\{|y(k)|^{2}\right\}=E\left\{\mathbf{w}^{H} \mathbf{X}(k) \mathbf{X}^{H}(k) \mathbf{w}\right\}=\mathbf{w}^{H} \mathbf{R}_{x x} \mathbf{w},
$$

where $E\{\cdot\}$ is an expected value, $\mathbf{R}_{x x}=E\left\{\mathbf{X}(k) \mathbf{X}^{H}(k)\right\}$ is the correlation matrix of the input signals.

Then the problem of finding optimum weight vector can be formulated as [5]:

$$
\begin{gathered}
\min _{\mathbf{w}} \mathbf{w}^{H} \mathbf{R}_{x x} \mathbf{w} \\
\text { subject to } \mathbf{C}^{H} \mathbf{w}=\mathbf{F}
\end{gathered}
$$

The optimum LCMV weight vector is: 


$$
\begin{gathered}
\mathbf{w}_{\text {opt }}=\mathbf{R}_{x x}^{-1} \mathbf{C}\left(\mathbf{C}^{H} \mathbf{R}_{x x}^{-1} \mathbf{C}\right)^{-1} \mathbf{F}, \\
\mathbf{C}=\left(\begin{array}{ccc}
\mathbf{c}_{0} & & \mathbf{0} \\
& \cdots & \\
\mathbf{0} & & \mathbf{c}_{0}
\end{array}\right),
\end{gathered}
$$

where $\tau_{i}=\tau_{i}(\theta, \varphi)$ is the propagation delay for the plane wave signal from the $0^{\text {th }}$ antenna to $i^{\text {th }}$ and $T_{s}$ is the delay between adjacent taps of the tapped delay lines.

where $\mathbf{C}$ is $M J \times r$ dimensional constraint matrix, $r$ is a number of constraints, $\mathbf{F}$ is $r$-dimensional response vector and $\mathbf{c}_{0}=\left(\begin{array}{lll}1 & \ldots & 1\end{array}\right)^{T}$ has dimension $M \times 1$. Thus, constraint matrix has a block-diagonal form.

In some applications, correlation matrix $\mathbf{R}_{x x}$ is unknown or may changes in time. In this case, we can use iterative algorithm for weight vector calculations. Therefore, we can replace correlation matrix by its simple approximation $\mathbf{R}_{x x}=\mathbf{X X} \mathbf{X}^{H}$. The iterative process can be written in the following form [5]:

$$
\mathbf{w}(k+1)=\mathbf{C}\left(\mathbf{C}^{H} \mathbf{C}\right)^{-1} \mathbf{F}+\mathbf{P}\left[\mathbf{w}(k)-\mu y^{*}(k) \mathbf{X}(k)\right],
$$

where $\mathbf{P}=\mathbf{E}-\mathbf{C}\left(\mathbf{C}^{H} \mathbf{C}\right)^{-1} \mathbf{C}^{H}, \mathbf{E}$ is the identity matrix, $\mu$ is scaling factor.

\section{DOA SIMPLE CONSTRAINTS}

Let us consider two elements adaptive antenna array with two tapped delay lines. Optimal weight coefficients are calculated by (4) or (6).

If the useful signal arrives from the broadside, the constraint matrix will have a form according to (5):

$$
\mathbf{C}_{n}=\left(\begin{array}{ll}
1 & 0 \\
1 & 0 \\
0 & 1 \\
0 & 1
\end{array}\right)
$$

In a case when the useful signal arrives from a direction different from the broadside the array can be steered mechanically or electrically [10]. This approach is not always appropriate in connection with practical implementation [8], [9].

To avoid this pre-steering let us rewrite constraint matrix (7) as the following:

$$
\mathbf{C}_{s}=\left(\begin{array}{cc}
\exp \left(-j \omega_{0} \tau_{0}\right) & 0 \\
\exp \left(-j \omega_{0} \tau_{1}\right) & 0 \\
0 & \exp \left[-j \omega_{0}\left(\tau_{0}+T_{s}\right)\right] \\
0 & \exp \left[-j \omega_{0}\left(\tau_{1}+T_{s}\right)\right]
\end{array}\right)
$$

\section{A. DOA is Broadside}

Assume that the useful signal arrives from the broadside then we can rewrite expression (8) in the following form:

$$
\mathbf{C}_{s}=\left(\begin{array}{cc}
1 & 0 \\
1 & 0 \\
0 & \exp \left(-j \omega_{0} T_{s}\right) \\
0 & \exp \left(-j \omega_{0} T_{s}\right)
\end{array}\right)
$$

taking into account that $\tau_{0}=\tau_{1}=0$ :

$$
\mathbf{C}_{s}=\mathbf{C}_{n}\left(\begin{array}{cc}
1 & 0 \\
0 & \exp \left(-j \omega T_{s}\right)
\end{array}\right)=\mathbf{C}_{n} \mathbf{C}_{0} .
$$

In (10) the second matrix is diagonal and unitary one:

$$
\mathbf{C}_{0}^{-1}=\mathbf{C}_{0}^{H}
$$

We consider expression $\mathbf{C}^{H} \mathbf{C}$ in (6):

$$
\mathbf{C}^{H} \mathbf{C}=M \mathbf{E},
$$

and we use constraint matrix (10):

$$
\mathbf{C}_{s}^{H} \mathbf{C}_{s}=M \mathbf{E},
$$

because matrix $\mathbf{C}_{0}$ is unitary one.

Now, we can rewrite matrix $\mathbf{P}$ in (6) with constraint matrix $\mathbf{C}_{s}$ :

$$
\mathbf{P}=\mathbf{E}-\frac{1}{M} \mathbf{C}_{s} \mathbf{C}_{s}^{H}
$$

We consider expression $\mathbf{C}_{s} \mathbf{C}_{s}^{H}$ in (14):

$$
\mathbf{C}_{s} \mathbf{C}_{s}^{H}=\mathbf{C}_{n} \mathbf{E} \mathbf{C}_{n}^{H}
$$

Finally, we get:

$$
\mathbf{P}=\mathbf{E}-\frac{1}{M} \mathbf{C}_{n} \mathbf{C}_{n}^{H}
$$

Moreover, we use response vector $\mathbf{F}$ in the following form: 


$$
F=\left(\begin{array}{l}
1 \\
0
\end{array}\right) .
$$

Therefore, the first term in (6) with constraint matrix (10) is rewritten in the following form:

$$
\mathbf{C}_{s}\left(\mathbf{C}_{s}{ }^{H} \mathbf{C}_{s}\right)^{-1} \mathbf{F} \equiv \frac{\mathbf{C}_{n} \mathbf{F}}{M} .
$$

Thus, optimal iterative solutions (6) are similar when we use constraint matrix (7) or (8) under the assumption that the useful signal arrives from the broadside. Next, we show that optimal direct solutions (4) are the similar too. We can rewrite (4) with constraint matrix (10):

$$
\mathbf{w}_{\text {opt } s}=\mathbf{R}_{x x}^{-1} \mathbf{C}_{n} \mathbf{C}_{0}\left(\mathbf{C}_{0}^{H} \mathbf{R}_{n n}^{-1} \mathbf{C}_{0}\right)^{-1} \mathbf{F},
$$

where $\mathbf{R}_{n n}^{-1}=\mathbf{C}_{n}^{H} \mathbf{R}_{x x}^{-1} \mathbf{C}_{n}$. form:

We can rewrite inversion of matrices in the following

$$
\left(\mathbf{C}_{0}^{-1} \mathbf{R}_{n n}^{-1} \mathbf{C}_{0}\right)^{-1}=\mathbf{C}_{0}^{-1} \mathbf{R}_{n n} \mathbf{C}_{0}
$$

Therefore, we can write $\mathbf{w}_{\text {opt }}$ in the following form:

$$
\mathbf{w}_{\text {opt } s}=\mathbf{R}_{x x}^{-1} \mathbf{C}_{n} \mathbf{R}_{n n} \mathbf{C}_{0} \mathbf{F},
$$

and taking into account that $\mathbf{R}_{n n}=\left(\mathbf{C}_{n}^{H} \mathbf{R}_{x x}^{-1} \mathbf{C}_{n}\right)^{-1}, \mathbf{C}_{0} \mathbf{F}=\mathbf{F}$ we get:

$$
\mathbf{w}_{\text {opts }}=\mathbf{R}_{x x}^{-1} \mathbf{C}_{n}\left(\mathbf{C}_{n}^{H} \mathbf{R}_{x x}^{-1} \mathbf{C}_{n}\right)^{-1} \mathbf{F} .
$$

As in the previous case, we can conclude that direct solutions in the form (4) are similar when we use constraint matrix (7) or (8) under the assumption that the useful signal arrives from the broadside.

\section{B. DOA is not Broadside}

Assume that the useful signal arrives from some direction except the broadside so we can rewrite expression (8) in the following form:

$$
\mathbf{C}_{s}=\mathbf{C}_{s 0} \mathbf{C}_{0},
$$

where

$$
\mathbf{C}_{s 0}=\left(\begin{array}{cc}
\exp \left(-j \omega_{0} \tau_{0}\right) & 0 \\
\exp \left(-j \omega_{0} \tau_{1}\right) & 0 \\
0 & \exp \left(-j \omega_{0} \tau_{0}\right) \\
0 & \exp \left(-j \omega_{0} \tau_{1}\right)
\end{array}\right)
$$

Let us consider the expressions for the product of matrices $\mathbf{C}_{s}^{H} \mathbf{C}_{s}$ and $\mathbf{C}_{s} \mathbf{C}_{s}^{H}$ that included in the formula (6) for calculating the optimal weighting coefficients:

$$
\begin{aligned}
& \mathbf{C}_{s}^{H} \mathbf{C}_{s}=2 \mathbf{E}, \\
& \mathbf{C}_{s 0}^{H} \mathbf{C}_{s 0}=\mathbf{E},
\end{aligned}
$$

$\mathbf{C}_{s 0}^{H} \mathbf{C}_{s 0}=\left(\begin{array}{cccc}e^{+j \omega_{0} \tau_{0}} & e^{+j \omega_{0} \tau_{1}} & 0 & 0 \\ 0 & 0 & e^{+j \omega_{0} \tau_{0}} & e^{+j \omega_{0} \tau_{1}}\end{array}\right)\left(\begin{array}{cc}e^{-j \omega_{0} \tau_{0}} & 0 \\ e^{-j \omega_{0} \tau_{1}} & 0 \\ 0 & e^{-j \omega_{0} \tau_{0}} \\ 0 & e^{-j \omega_{0} \tau_{1}}\end{array}\right)=2 \mathbf{E}$.

In a common case, we can write $\mathbf{C}_{s}^{H} \mathbf{C}_{s}=M \mathbf{E}$.

The second product has a form:

$$
\mathbf{C}_{s} \mathbf{C}_{s}^{H}=\mathbf{C}_{s 0} \mathbf{C}_{s 0}^{H} .
$$

Now, we can rewrite expression for matrix P in (6) in the following form:

$$
\mathbf{P}=\mathbf{E}-\frac{1}{M} \mathbf{C}_{s 0} \mathbf{C}_{s 0}^{H} .
$$

The first term in (6) will have a form:

$$
\mathbf{C}_{s}\left(\mathbf{C}_{s}{ }^{H} \mathbf{C}_{s}\right)^{-1} \mathbf{F} \equiv \frac{\mathbf{C}_{s 0} \mathbf{F}}{M} .
$$

We can rewrite iterative solution (6) with constraint matrix (24):

$$
\mathbf{w}(k+1)=\frac{\mathbf{C}_{s 0} \mathbf{F}}{M}+\mathbf{P}\left[\mathbf{w}(k)-\mu y^{*}(k) \mathbf{X}(k)\right] .
$$

From the obtained expressions it can be concluded that constraint matrix $\mathbf{C}_{s 0}$ (24) can be used instead matrix $\mathbf{C}_{s}$ (23). (23):

Next, we write direct solution (4) with constraint matrix

$$
\mathbf{w}_{\text {opt } s 0}=\mathbf{R}_{x x}^{-1} \mathbf{C}_{s 0} \mathbf{C}_{0}\left(\mathbf{C}_{0}^{H} \mathbf{R}_{s s}^{-1} \mathbf{C}_{0}\right)^{-1} \mathbf{F},
$$


where $\mathbf{R}_{s s}^{-1}=\mathbf{C}_{s 0}^{H} \mathbf{R}_{x x}^{-1} \mathbf{C}_{s 0}$.

We can rewrite inversion of matrices in the following form:

$$
\left(\mathbf{C}_{0}^{-1} \mathbf{R}_{s s}^{-1} \mathbf{C}_{0}\right)^{-1}=\mathbf{C}_{0}^{-1} \mathbf{R}_{s s} \mathbf{C}_{0}
$$

Therefore, we can write $\mathbf{w}_{\text {opt } s 0}$ in the following form:

$$
\mathbf{w}_{\text {opt } s 0}=\mathbf{R}_{x x}^{-1} \mathbf{C}_{s 0} \mathbf{R}_{s s} \mathbf{C}_{0} \mathbf{F},
$$

and taking into account that $\mathbf{R}_{s s}=\left(\mathbf{C}_{s 0}^{H} \mathbf{R}_{x x}^{-1} \mathbf{C}_{s 0}\right)^{-1}$ and $\mathbf{C}_{0} \mathbf{F}=\mathbf{F}$ we get:

$$
\mathbf{w}_{\text {opt } s 0}=\mathbf{R}_{x x}^{-1} \mathbf{C}_{s 0}\left(\mathbf{C}_{s 0}^{H} \mathbf{R}_{x x}^{-1} \mathbf{C}_{s 0}\right)^{-1} \mathbf{F}
$$

As we can see when we use different constraint matrices (23) and (24) similar solutions are obtained. Therefore, we can use constraint matrix (24) to make DOA constraints.

\section{Simulation RESUltS}

As an example, we consider uniform linear antenna array of four isotropic elements and four tapped delay lines. Therefore, we can show that results obtained in Section III can be applied to arrays with number of elements more than two. Distance between adjacent elements is $\lambda / 2, \lambda$ is wavelength. We assume that useful signal DOA is $30^{\circ}$ and interference signal DOA is $-60^{\circ}$.

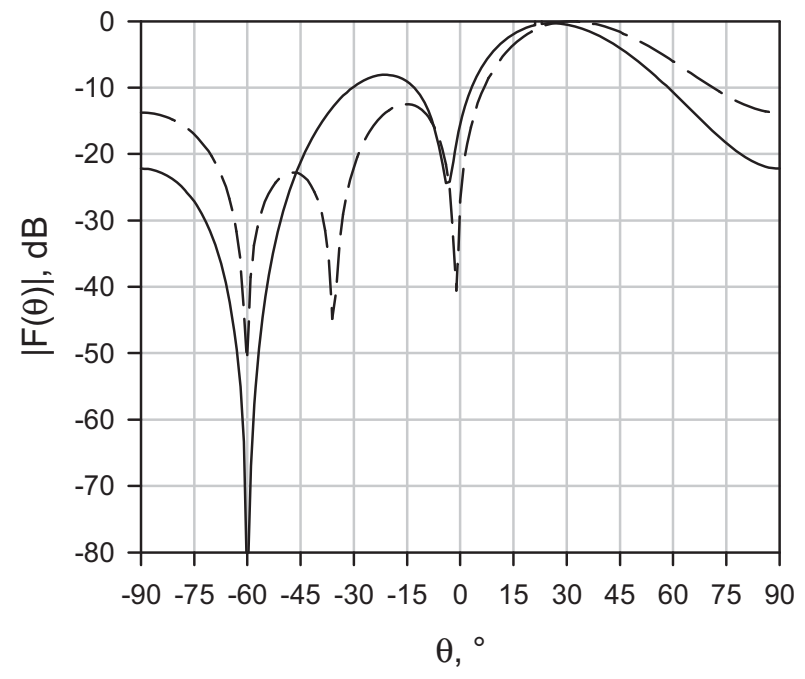

Fig. 2. Radiation patterns

Fig. 2 shows radiation patterns are obtained by direct solution (34) (solid line) and iterative solution (30) (dash line). As we can see, main lobes are orient in useful signal DOA.

\section{CONCLUSION}

It is shown that we do not need to use pre-steering with additional delay lines in the case where useful signal arrives from an arbitrary direction. It is enough to use pre-steered simple constraint matrix.

In the case where DOA is the broadside, it was possible to simplify the iterative Frost's solution. Moreover, constraints in the form (8) are equivalent to the constraints in the form (7). In addition, the direct solution of an LCMVproblem with constraints in the form (8) is equivalent to the solution with constraints in the form (7).

The use of constraints in the form (8) is unnecessary for an arbitrary DOA of the signal. It suffices to use constraints in the form (24).

It should be noted that the obtained results can be applied to an adaptive array with any number of elements.

\section{REFERENCES}

[1] R. L. Fante and J. J. Vaccaro, "Wideband cancellation of interference in a GPS receive array," IEEE Transactions on Aerospace and Electronic Systems, vol. 36, no. 2, pp. 549-564, April 2000.DOI: 10.1109/7.845241

[2] M Rasekh and S. R. Seydnejad, "Design of an adaptive wideband beamforming algorithm for conformal arrays," IEEE Communications Letters, vol. 18, no. 11, pp. 1955-1958, November 2014. DOI: 10.1109/LCOMM.2014.2357417

[3] A. El-Keyi and B. Champagne, "Adaptive linearly constrained minimum variance beamforming for multiuser cooperative relaying using the kalman filter," IEEE Transactions on Wireless Communications, vol. 9, no. 2, pp. 641-651, February 2010. DOI: 10.1109/TWC.2010.02.081636

[4] J. Dmochowski, J. Benesty and S. Affes, "Linearly Constrained Minimum Variance Source Localization and Spectral Estimation," IEEE Transactions on Audio, Speech, and Language Processing, vol. 16, no. 8, pp. 1490-1502, November 2008. DOI: 10.1109/TASL.2008.2005029

[5] O.L. Frost, "An algorithm for linearly constrained adaptive array processing," Proceedings of the IEEE, vol. 60, no. 8, pp. 926-935, August 1972. DOI: 10.1109/PROC.1972.8817

[6] A. El-Keyi, T. Kirubarajan and A.B. Gershman, "Wideband robust beamforming based on worst-case performance optimization," 2005 IEEE/SP 13th Workshop on Statistical Signal Processing, July 2005. DOI: $10.1109 /$ SSP.2005.1628604

[7] K. Takao, M. Fujita and T. Nishi, "An adaptive antenna array under directional constraint," IEEE Transactions on Antennas and Propagation, vol. 24, no. 5, pp. 662-669, September 1976. DOI: 10.1109/TAP.1976.1141411

[8] R. Ebrahimi and S. R. Seydnejad, "Elimination of pre-steering delays in space-time broadband beamforming using frequency domain constraints," IEEE Communications Letters, vol. 17, no. 4, pp. 769772, April 2013. DOI: 10.1109/LCOMM.2013.022713.130090

[9] L.C. Godara and M.R. Sayyah Jahromi, "New constraints for broadband beamformers without steering delays," 2005. IEEE/ACES International Conference on Wireless Communications and Applied Computational Electromagnetics, April 2005. DOI: 10.1109/WCACEM.2005.1469746

[10] W. Liu and S. Weiss, Wideband Beamforming: Concepts and Techniques. Wiley, 2010 